\title{
ASYMPTOTIC FORMULA FOR THE SOLUTION OF THE STOKES PROBLEM WITH A SMALL PERTURBATION OF THE DOMAIN IN TWO AND THREE DIMENSIONS
}

\author{
THI HONG CAM LUONG AND CHRISTIAN DAVEAU
}

\begin{abstract}
In this paper we consider the resolvent Stokes problem in the case there is a small perturbation of the domain caused by a perturbed boundary. Firstly, we prove that the solution of Stokes problem is continuous due to this small perturbation. Secondly, we derive the first-order term in the displacement field perturbation that due to the deformation of the domain. It is worth emphasizing that even though only the first-order term is given, our method enables us to derive higher-order terms as well. The derivation is rigorous and based on layer potential techniques.
\end{abstract}

\section{Contents}

1. Introduction

2. Definitions and Preliminary results

2.1. Small perturbation of an interface

2.2. The potential theory for Stokes resolvent system

3. Continuity of the solution with respect to the perturbation

4. Derivation of the asymptotic expansion

References

\section{INTRODUCTION}

Let $\Omega \subset \mathbb{R}^{d}(d=2,3)$ be a bounded domain with boundary $\partial \Omega$ of class $\mathcal{C}^{2}$. We consider the Stokes resolvent system

$$
\left\{\begin{array}{lll}
-\Delta u+\nabla p=\lambda u & \text { in } & \Omega \\
\nabla \cdot u=0 & \text { in } & \Omega \\
u=g & \text { on } & \partial \Omega .
\end{array}\right.
$$

with $u(x)$ is the velocity of the fluid, $p(x)$ is the pressure, and $g(x)$ is the boundary condition.

Denote by $\nu$ the outward unit normal to $\partial \Omega$, let $\partial \Omega_{\delta}$ be a $\delta$-perturabation of $\partial \Omega$ as follows:

In the two-dimensional case, let $\rho \in \mathcal{C}^{1}(\partial \Omega)$, we consider the perturbed boundary $\partial \Omega_{\delta}$ be given by

$$
\partial \Omega_{\delta}=\{\widetilde{x}: \widetilde{x}=x+\delta \rho(x) \nu(x), x \in \partial \Omega\}
$$

Date: April 8, 2013.

2010 Mathematics Subject Classification. 45B05. 
and in the three-dimensional case, we consider the parallel surface

$$
\partial \Omega_{\delta}=\{\widetilde{x}: \widetilde{x}=x+\delta \nu(x), x \in \partial \Omega\} .
$$

Here we consider the parameter $\delta>0$ which tends to 0 . We assume that $g$ is an analytic function in a small neighborhood of $\partial \Omega$, and denote by $g_{\delta}$ the extended function of $g$ onto $\partial \Omega_{\delta}$. Then denote by $u_{\delta}$ the solution of the perturbation problem

$$
\left\{\begin{array}{lll}
-\Delta u_{\delta}+\nabla p_{\delta}=\lambda u_{\delta} & \text { in } & \Omega_{\delta} \\
\nabla \cdot u_{\delta}=0 & \text { in } & \Omega_{\delta} \\
u_{\delta}=g_{\delta} & \text { on } & \partial \Omega_{\delta} .
\end{array}\right.
$$

In this paper we verify the continuity of the solution $u_{\delta}$ with respect to $\delta$ and derive the asymptotic expansion of $\left.\left(u_{\delta}-u\right)\right|_{\Omega^{\circ}}$ as $\delta$ tends to 0 , where $\Omega^{\circ}$ is any closed subset of $\Omega \cap \Omega_{\delta}$. The asymptotic expansion is derived due to the theory of layer potentials and Fredholm's alternative, and the properties of small perturbation of an interface. In connection with this, we refer to recent works in the context of interface problems [1], [2], [7], the continuity of the solution due to a small perturbation of an interface [7] and layer-potential theory for Stokes problem [3], [4], [5], [6], [8], [9]. In the work of H. Ammari, H. Kang, M. Lim, and H. Zribi [1] , they derived the asymptotic expansion of the boundary perturbations of steady-state voltage potentials, and now in our work, we derive the asymptotic expansion for the Stokes problem with Dirichlet boundary condition. However, by the same method, we can derive asymptotic formula for the Neumann problem as well.

This paper is organized as follows. In the next section, we introduce some notations for small perturbations of an interface of class $\mathcal{C}^{2}$, review some basic facts on the layer-potentials and give representation formulas. In Section 3, we verify the continuity of the solution with respect to $\delta$, and in Section 4 , we derive asymptotic expansion for the displacement field perturbation in term of $\delta$.

\section{Definitions and Preliminary Results}

\subsection{Small perturbation of an interface.}

2.1.1. In the case $d=2$. Let $a, b \in \mathbb{R}$, with $a<b$, and let $X(t):[a, b] \rightarrow \mathbb{R}^{2}$ be the arclength parametrization of $\partial \Omega$, namely, $X$ is a $\mathcal{C}^{2}$-function satisfying $\left|X^{\prime}(t)=1\right|$ for all $t \in[a, b]$ and

$$
\partial \Omega:=\{x=X(t), t \in[a, b]\} .
$$

Then the outward unit normal to $\partial \Omega, \nu(x)$, is given by $\nu(x)=R_{-\pi} X^{\prime}(t)$, where $R_{\frac{-\pi}{2}}$ is the rotation by $-\pi / 2$; the tangential vector at $x, T(x)=X^{\prime}(t)$, and $X^{\prime}(t) \perp X^{\prime \prime}(t)$. Set the curvature $\tau(x)$ to be defined by

$$
X^{\prime \prime}(t)=\tau(x) \nu(x) .
$$

We will sometimes use $\rho(x)$ for $\rho(X(t))$ and $\rho^{\prime}(x)$ for the tangential derivative of $\rho(x)$. 
Then, $\widetilde{x}=\widetilde{X}(t)=X(t)+\delta \rho(x) \nu(x)=X(t)+\delta \rho(x) R_{\frac{-\pi}{2}} X^{\prime}(t)$ is a parametrization of $\partial \Omega_{\delta}$. We denote by $\widetilde{\nu}(\widetilde{x})$ the outward unit normal to $\partial \Omega_{\delta}$ at $\widetilde{x}$. Then, it is proved in [1] that $\widetilde{\nu}(\widetilde{x})$ can be expanded uniformly as

$$
\widetilde{\nu}(\widetilde{x})=\sum_{n=0}^{\infty} \delta^{n} \nu^{n}(x), \text { with } \widetilde{x}=x+\delta \rho(x) \nu(x), \widetilde{x} \in \partial \Omega_{\delta}, x \in \partial \Omega,
$$

where the vector-valued functions $\nu^{n}(x)$ are uniformly bounded regardless of $n$. In particular,

$$
\nu^{0}(x)=\nu(x), \nu^{1}(x)=-\rho(x) T(x), \quad x \in \partial \Omega .
$$

Likewise, denote by $d \sigma_{\delta}(\widetilde{x})$ the length element of $\partial \Omega_{\delta}$ at $\widetilde{x}$, which has an uniform expansion (see in [1])

$$
d \sigma_{\delta}(\widetilde{x})=\left|\widetilde{X}^{\prime}(t)\right| d t=\sqrt{(1-\delta \tau(t) \rho(t))^{2}+\delta^{2} \rho^{\prime 2}(t)} d t=\sum_{n=0}^{\infty} \delta^{n} \sigma^{n}(x) d \sigma(x),
$$

with $\widetilde{x} \in \partial \Omega_{\delta}, x \in \partial \Omega$, and $\sigma^{n}$ are functions bounded regardless of $n$, with

$$
\sigma^{0}(x)=1, \sigma^{1}(x)=-\tau(x) \rho(x), x \in \partial \Omega .
$$

Let $\widetilde{x}, \widetilde{y} \in \partial \Omega_{\delta}$, that is $\widetilde{x}=x+\delta \rho(x) \nu(x), \widetilde{y}=y+\delta \rho(y) \nu(y)$, then

$$
\widetilde{x}-\widetilde{y}=x-y+\delta(\rho(x) \nu(x)-\rho(y) \nu(y)),
$$

and

$|\widetilde{x}-\widetilde{y}|^{2}=|x-y|^{2}\left(1+2 \delta \frac{\langle x-y, \rho(x) \nu(x)-\rho(y) \nu(y)\rangle}{|x-y|^{2}}+\delta^{2} \frac{|\rho(x) \nu(x)-\rho(y) \nu(y)|^{2}}{|x-y|^{2}}\right)$.

Denote by

$$
E(x, y):=\frac{\langle x-y, \rho(x) \nu(x)-\rho(y) \nu(y)\rangle}{|x-y|^{2}}, G(x, y):=\frac{|\rho(x) \nu(x)-\rho(y) \nu(y)|^{2}}{|x-y|^{2}} .
$$

Since $\partial \Omega$ is of class $\mathcal{C}^{2}$, we can see that

$$
|E(x, y)|+|G(x, y)|^{\frac{1}{2}} \leq C\|X\|_{\mathcal{C}^{2}(\partial \Omega)}\|\rho\|_{\mathcal{C}^{1}(\partial \Omega)} \text {, for all } x, y \in \partial \Omega,
$$

and hence

$$
|\widetilde{x}-\widetilde{y}|=|x-y| \sqrt{1+2 \delta E(x, y)+\delta^{2} G(x, y)}=|x-y| \sum_{n=0}^{\infty} \delta^{n} L_{n}(x, y),
$$

where the series converges absolutely and uniformly. In particular, we can see that

$$
L_{0}(x, y)=1, L_{1}(x, y)=E(x, y) .
$$


2.1.2. In the case $d=3$. Denote by $d \sigma_{\delta}(\widetilde{x})$ the surface element of $\partial \Omega_{\delta}$ at $\widetilde{x}$. Thanks to the results in [2] we get the following uniform expansion for $d \sigma_{\delta}(\widetilde{x})$

$$
d \sigma_{\delta}(\widetilde{x})=\sum_{n=0}^{\infty} \delta^{n} \sigma^{n}(x) d \sigma(x), \text { with } \widetilde{x}=x+\delta \nu(x), \quad \widetilde{x} \in \partial \Omega_{\delta}, x \in \partial \Omega .
$$

where $\sigma^{n}$ are functions bounded regardless of $n$, with

$$
\sigma^{0}(x)=1, \sigma^{1}(x)=-2 H(x), \sigma^{2}(x)=K(x), x \in \partial \Omega,
$$

with $H$ and $K$ denote the mean and Gaussian curvature of $\partial \Omega$ respectively. In the other hand, due to the parallel property of $\partial \Omega$ and $\partial \Omega_{\delta}$ we have

$$
\widetilde{\nu}(\widetilde{x})=\nu(x), \text { with } \widetilde{x}=x+\delta \nu(x), \widetilde{x} \in \partial \Omega_{\delta}, x \in \partial \Omega .
$$

Let $\widetilde{x}, \widetilde{y} \in \partial \Omega_{\delta}$, then $\widetilde{x}-\widetilde{y}=x-y+\delta(\nu(x)-\nu(y))$, and

$$
|\widetilde{x}-\widetilde{y}|^{2}=|x-y|^{2}\left(1+2 \delta \frac{\langle x-y, \nu(x)-\nu(y)\rangle}{|x-y|^{2}}+\delta^{2} \frac{|\nu(x)-\nu(y)|^{2}}{|x-y|^{2}}\right) .
$$

Denote by

$$
E(x, y):=\frac{\langle x-y, \nu(x)-\nu(y)\rangle}{|x-y|^{2}}, G(x, y):=\frac{|\nu(x)-\nu(y)|^{2}}{|x-y|^{2}} .
$$

Since $\partial \Omega$ is of class $\mathcal{C}^{2}$, we can see that there exists a constant $C$ depending only on $\partial \Omega$ such that

$$
|E(x, y)|+|G(x, y)|^{\frac{1}{2}} \leq C, \text { for all } x, y \in \partial \Omega,
$$

and hence

$$
|\widetilde{x}-\widetilde{y}|=|x-y| \sqrt{1+2 \delta E(x, y)+\delta^{2} G(x, y)}=|x-y| \sum_{n=0}^{\infty} \delta^{n} L_{n}(x, y),
$$

where the series converges absolutely and uniformly. In particular, we can see that

$$
L_{0}(x, y)=1, L_{1}(x, y)=E(x, y) .
$$

2.2. The potential theory for Stokes resolvent system. We start to review some basic facts in the theory of layer potentials. We consider here $\lambda \in \mathbb{C} \backslash\{z \in \mathbb{C}: \operatorname{Re} z \leq 0, \operatorname{Im} z=0\}$. The fundamental tensors $(\Gamma, F)$ of the Stokes resolvent system (1.1) can be obtained by the Fourier transform method in the following forms (see [3] for $d=2$ and [4], [8] for $d=3$ ):

In two dimensions, we have

$$
\left\{\begin{array}{l}
\Gamma_{i j}(x)=-\frac{1}{2 \pi}\left\{\delta_{i j} e_{1}(\sqrt{\lambda}|x|)+\frac{x_{i} x_{j}}{|x|^{2}} e_{2}(\sqrt{\lambda}|x|)\right\}, \\
F_{i}(x)=-\frac{x_{i}}{2 \pi|x|^{2}}
\end{array}\right.
$$

for $\forall x \in \mathbb{R}^{2}, x \neq 0$ with

$$
\begin{gathered}
e_{1}(\kappa)=K_{0}(\kappa)+\kappa^{-1} K_{1}(\kappa)-\kappa^{-2} \\
e_{2}(\kappa)=-K_{0}(\kappa)-2 \kappa^{-1} K_{1}(\kappa)+2 \kappa^{-2},
\end{gathered}
$$

where $K_{n}\left(n \in \mathbb{N}_{0}\right)$ denotes the modified Bessel function of order $n$, and $\delta_{i j}$ is the Kronecker'symbol. 
In three dimensions, we have

$$
\left\{\begin{array}{l}
\Gamma_{i j}(x)=-\frac{1}{4 \pi}\left\{\delta_{i j} e_{1}(-\sqrt{\lambda}|x|)+\frac{x_{i} x_{j}}{|x|^{3}} e_{2}(-\sqrt{\lambda}|x|)\right\}, \\
F_{i}(x)=-\frac{1}{4 \pi} \frac{x_{i}}{|x|^{3}}
\end{array}\right.
$$

for $\forall x \in \mathbb{R}^{3}, x \neq 0$ with

$$
\begin{gathered}
e_{1}(\epsilon)=\sum_{n=0}^{\infty} \frac{(n+1)^{2}}{(n+2) !} \epsilon^{n}=\exp (\epsilon)\left(1-\epsilon^{-1}+\epsilon^{-2}\right)-\epsilon^{-2} \\
e_{2}(\epsilon)=\sum_{n=0}^{\infty} \frac{1-n^{2}}{(n+2) !} \epsilon^{n}=\exp (\epsilon)\left(-1+3 \epsilon^{-1}-3 \epsilon^{-2}\right)+3 \epsilon^{-2} .
\end{gathered}
$$

By using the notations $\hat{x}=x-y=\left(\hat{x}_{1}, \cdots, \hat{x}_{d}\right)$ and $r=|\hat{x}|$, we introduce the stress tensor $\mathcal{S}$ associated to the fundamental tensors $(\Gamma, F)$ and having the following components (see in [8]):

$$
S_{i j k}(x, y):=-F_{j}(\hat{x}) \delta_{i k}+\frac{\partial \Gamma_{i j}(\hat{x})}{\partial \hat{x}_{k}}+\frac{\partial \Gamma_{k j}(\hat{x})}{\partial \hat{x}_{i}}, i, j, k=1, \cdots, d .
$$

Note that we used the Einstein convention for the summation notation omitting the summation sign for the indices appearing twice. We will continue using this convention throughout this paper. Taking into account the relation (2.8) and (2.9) we obtain the following explicit forms (see in [8]) as follows.

In two dimensions, for $\forall x, y \in \mathbb{R}^{2}, x \neq y$ we have:

with

$$
\begin{aligned}
S_{i j k}(x, y)=-\frac{1}{2 \pi}\left\{\delta_{i k} \frac{\hat{x}_{j}}{r^{2}}\right. & \left.d_{1}(\sqrt{\lambda} r)+\left(\delta_{k j} \frac{\hat{x}_{i}}{|x|^{2}}-\delta_{i j} \frac{\hat{x}_{k}}{r^{2}}\right) d_{2}(\sqrt{\lambda} r)\right\} \\
-\frac{1}{2 \pi} & \left\{\frac{\hat{x}_{i} \hat{x}_{j} \hat{x}_{k}}{r^{4}}\left(2 d_{1}(\sqrt{\lambda} r)+2 d_{2}(\sqrt{\lambda} r)-2\right)\right\},
\end{aligned}
$$

with

$$
d_{1}(\kappa)=2 K_{2}(\kappa)+1-4 \kappa^{-2}, d_{2}(\kappa)=2 K_{2}(\kappa)+\kappa K_{1}(\kappa)-4 \kappa^{-2} .
$$

In three dimensions, for $\forall x, y \in \mathbb{R}^{3}, x \neq y$, we have:

$$
\begin{aligned}
S_{i j k}(x, y)=-\frac{1}{4 \pi}\left\{\delta_{i k} \frac{\hat{x}_{j}}{r^{3}}\right. & \left.d_{1}(-\sqrt{\lambda} r)-\left(\delta_{i j} \frac{\hat{x}_{k}}{r^{3}}+\delta_{k j} \frac{\hat{x}_{i}}{r^{3}}\right) d_{2}(-\sqrt{\lambda} r)\right\} \\
- & \frac{1}{4 \pi}\left\{\frac{\hat{x}_{i} \hat{x}_{j} \hat{x}_{k}}{r^{5}}\left(3-3 d_{1}(-\sqrt{\lambda} r)+2 d_{2}(-\sqrt{\lambda} r)\right)\right\},
\end{aligned}
$$

$$
\begin{aligned}
& d_{1}(\epsilon)=\sum_{n=2}^{\infty} \frac{2\left(n^{2}-1\right)}{(n+2) !} \epsilon^{n}=\exp (\epsilon)\left(2-6 \epsilon^{-1}+6 \epsilon^{-2}\right)-6 \epsilon^{-2}+1, \\
& d_{2}(\epsilon)=\sum_{n=2}^{\infty} \frac{n\left(n^{2}-1\right)}{(n+2) !} \epsilon^{n}=\exp (\epsilon)\left(\epsilon-3+6 \epsilon^{-1}-6 \epsilon^{-2}\right)+6 \epsilon^{-2} .
\end{aligned}
$$

The pressure tensor $\Lambda$ associated to the stress tensor $\mathcal{S}$ has the following components (see in [3] for $d=2$ and [4], [5] for $d=3$ ):

$$
\Lambda_{i k}(x, y)= \begin{cases}-\frac{1}{2 \pi}\left(\delta_{i k} \lambda \ln r-4 \frac{\delta_{i k}}{r^{2}}+8 \frac{\hat{x}_{i} \hat{x}_{k}}{r^{4}}\right) & \text { for } d=2 \\ -\frac{1}{4 \pi}\left(\frac{\delta_{i k}}{r^{3}}\left(\lambda r^{2}-2\right)+\frac{\hat{x}_{i} \hat{x}_{k}}{r^{5}}\right) & \text { for } d=3,\end{cases}
$$


for $\forall x, y \in \mathbb{R}^{d}, x \neq y$.

Next we consider the single- and double-layer potentials associcated with stress and pressure tensors. Let $\partial D$ be a closed surface of class $C^{2}$ of a bounded domain $D$ and $\phi=\left(\phi_{1}, \cdots, \phi_{d}\right)$ a vectorial continuous function on $\partial D$. For $x \in \mathbb{R}^{d} \backslash \partial D$, we define the single-layer potential $\mathcal{V}_{D} \phi$ which has components as follows:

$$
\mathcal{V}_{D}^{i} \phi(x):=\int_{\partial D} \Gamma_{i j}(x-y) \phi_{j}(y) d \sigma(y), i=1, \cdots, d ;
$$

and the double-layer potential $\mathcal{W}_{D} \phi$ has components as follows

$$
\mathcal{W}_{D}^{i} \phi(x):=\int_{\partial D}-S_{i j k}(x, y) \nu_{k}(y) \phi_{j}(y) d \sigma(y), i=1, \cdots, d ;
$$

where $\nu(y)$ is the outward unit normal vector to $\partial D$ at the point $y$.

Additionally, we consider the functions $\mathcal{Q}_{D} \phi$ and $\Pi_{D} \phi$ as follows

$$
\begin{aligned}
& \mathcal{Q}_{D} \phi(x):=\int_{\partial D} F_{i}(x-y) \phi_{i}(y) d \sigma(y), \\
& \Pi_{D} \phi(x):=\int_{\partial D} \Lambda_{i k}(x-y) \nu_{k}(y) \phi_{i}(y) d \sigma(y)
\end{aligned}
$$

for $x \in \mathbb{R}^{d} \backslash \partial D$.

The functions $\left(\mathcal{V}_{D} \phi, \mathcal{Q}_{D} \phi\right)$ and $\left(\mathcal{W}_{D} \phi, \Pi_{D} \phi\right)$ are smooth functions in each of the domains $\mathbb{R}^{d} \backslash \bar{D}_{0}$ and $D_{0}$, respectively, where $D_{0}$ is the inner domain with the boundary $\partial D$. All these functions satisfy the following equations of Stokes resolvent problem:

$$
\begin{aligned}
& -\Delta \mathcal{V}_{D} \phi(x)+\nabla \mathcal{Q}_{D} \phi(x)=0, \quad \nabla \cdot \mathcal{V}_{D} \phi(x)=0 \\
& -\Delta \mathcal{W}_{D} \phi(x)+\nabla \Pi_{D} \phi(x)=0, \quad \nabla \cdot \Pi_{D} \phi(x)=0
\end{aligned}
$$

for $x \in \mathbb{R}^{3} \backslash \partial D$.

For our prupose, we also need to introcduce the principal value of the doublelayer potential in a point $x_{0}$ of $\partial D$ defined by the following formula

$$
\mathcal{K}_{D}^{i} \phi\left(x_{0}\right):=p . v \cdot \int_{\partial D}-S_{i j k}\left(x_{0}, y\right) \nu_{k}(y) \phi_{j}(y) d \sigma(y)
$$

For $\phi \in(C(\partial D))^{d}$, the following trace relation for $\mathcal{W}_{D}$ holds (see in [6]):

$$
\left.\mathcal{W}_{D} \phi\right|_{ \pm}=\left(\mp \frac{1}{2} I+\mathcal{K}_{D}\right) \phi \quad \text { a.e. on } \partial \Omega
$$

where $\left.\mathcal{W}_{D} \phi\right|_{-}$and $\left.\mathcal{W}_{D} \phi\right|_{+}$denote the limits from inside $D$ and outside $D$.

From the previous section, the solution for the interior Dirichlet problem can be presented by the pure double-layer potential (see in [4]), then taking $D=\Omega$ and $D=\Omega_{\delta}$ in the fomulae (2.13), (2.14), (2.15) and (2.17), we get the boundary integral representation of the solutions for the initial Stokes problem (1.1) and the perturbation problem (1.2) are $\left(\mathcal{W}_{\Omega} \phi, \Pi_{\Omega} \phi\right)$ and $\left(\mathcal{W}_{\Omega_{\delta}} \widetilde{\phi}, \Pi_{\Omega_{\delta}} \widetilde{\phi}\right)$, respectively. 
Additionally, from (2.10) and (2.11), we conclude that the components $S_{i j k}(x, y)$ $\nu_{k}(y)$ of $\mathcal{S}(x, y) \nu(y)$ can be written in the following manner (see in [3],[4]).

In two dimensions, for $\forall x, y \in \mathbb{R}^{2}, x \neq y$, we have

$$
\begin{array}{r}
\mathcal{S}_{i j k}(x, y) \nu_{k}(y)=-\frac{1}{2 \pi}\left\{\frac{r_{i} \nu_{j}}{|r|^{2}} d_{1}(\sqrt{\lambda}|r|)+\left(\frac{\nu_{i} r_{j}}{|r|^{2}}-\delta_{i j} \frac{r \cdot \nu}{|r|^{2}}\right) d_{2}(\sqrt{\lambda}|r|)\right\}, \\
-\frac{1}{2 \pi}\left\{\frac{r_{i} r_{j} r \cdot \nu}{|r|^{4}}\left(2 d_{1}(\sqrt{\lambda}|r|)+2 d_{2}(\sqrt{\lambda}|r|)-2\right)\right\}
\end{array}
$$

with

$$
d_{1}(\kappa)=2 K_{2}(\kappa)+1-4 \kappa^{-2}, d_{2}(\kappa)=2 K_{2}(\kappa)+\kappa K_{1}(\kappa)-4 \kappa^{-2} .
$$

And in three dimensions, for $\forall x, y \in \mathbb{R}^{3}, x \neq y$, we have

$$
\begin{aligned}
\mathcal{S}_{i j k}(x, y) \nu_{k}(y)=-\frac{1}{4 \pi} & \left\{\frac{r_{i} \nu_{j}}{|r|^{3}} d_{1}(-\sqrt{\lambda}|r|)-\left(\frac{\nu_{i} r_{j}}{|r|^{3}}+\delta_{i j} \frac{r \cdot \nu}{|r|^{3}}\right) d_{2}(-\sqrt{\lambda}|r|)\right\} \\
& -\frac{1}{4 \pi}\left\{\frac{r_{i} r_{j} r \cdot \nu}{|r|^{5}}\left(3-3 d_{1}(-\sqrt{\lambda}|r|)+2 d_{2}(-\sqrt{\lambda}|r|)\right)\right\},
\end{aligned}
$$

with

$$
\begin{aligned}
& d_{1}(\epsilon)=\sum_{n=2}^{\infty} \frac{2\left(n^{2}-1\right)}{(n+2) !} \epsilon^{n}=\exp (\epsilon)\left(2-6 \epsilon^{-1}+6 \epsilon^{-2}\right)-6 \epsilon^{-2}+1 \\
& d_{2}(\epsilon)=\sum_{n=2}^{\infty} \frac{n\left(n^{2}-1\right)}{(n+2) !} \epsilon^{n}=\exp (\epsilon)\left(\epsilon-3+6 \epsilon^{-1}-6 \epsilon^{-2}\right)+6 \epsilon^{-2} .
\end{aligned}
$$

For further purpose, we denote the kernel $-S_{i j k}(x, y) \nu_{k}(y)$ of double-layer potential $\mathcal{K}_{D} \phi$ by $\mathcal{D}_{i j}(x, y)$. We note that $\mathcal{D}_{i j}(x, y)$ behaves like $\mathcal{O}\left(r^{-d+1}\right)$ as $r \rightarrow 0$, which implies this kernel is weakly singular, so the integral operator is bounded on $(\mathcal{C}(\partial D))^{d}$ (see in [11, p. 243] or in [3], [4], [6]).

\section{Continuity of the solution with Respect to the Perturbation}

In this section we will show that the solution $u_{\delta}$ of the perturbation problem (1.2) is continuous with respect to $\delta$ as $\delta$ tends to 0 . And for the guarantee of the solvability and uniqueness of the solutions for (1.1) and (1.2), we assume the following compatibility conditions

$$
\int_{\partial \Omega} g \cdot n d \sigma=0, \text { and } \int_{\partial \Omega_{\delta}} g_{\delta} \cdot n_{\delta} d \sigma_{\delta}=0 .
$$

From the previous section, the solution to the Stokes system (1.1) can be represented by the pure double-layer potential on $\partial \Omega$

$$
u^{i}(x)=\mathcal{W}_{\Omega}^{i} \phi(x)=\int_{\partial \Omega}-S_{i j k}(x, y) \nu_{k}(y) \phi_{i}(y) d \sigma(y), x \in \Omega
$$

with the density vectorial function $\phi$ satisfies

$$
\left.\mathcal{W}_{\Omega} \phi\right|_{-}=\left(\frac{1}{2} I+\mathcal{K}_{\Omega}\right) \phi \quad \text { a.e. on } \partial \Omega .
$$


Likewise, the solution to the Stokes perturbation problem (1.2) can be represented by the pure double-layer potential on $\partial \Omega_{\delta}$

$$
u_{\delta}^{i}(x)=\mathcal{W}_{\Omega_{\delta}}^{i} \widetilde{\phi}(x)=\int_{\partial \Omega_{\delta}}-S_{i j k}(x, y) \nu_{k}(y) \widetilde{\phi}_{i}(y) d \sigma(y), x \in \Omega_{\delta},
$$

with the density vectorial function $\widetilde{\phi}$ satisfies

$$
\left.\mathcal{W}_{\Omega_{\delta}} \widetilde{\phi}\right|_{-}=\left(\frac{1}{2} I+\mathcal{K}_{\Omega_{\delta}}\right) \widetilde{\phi} \quad \text { a.e. on } \partial \Omega_{\delta} .
$$

From the boundary condition we have $\left.\mathcal{W}_{\Omega} \phi\right|_{-}=g$ on $\partial \Omega$ and $\left.\mathcal{W}_{\Omega_{\delta}} \widetilde{\phi}\right|_{-}=g_{\delta}$ on $\partial \Omega_{\delta}$. Consider $u_{\delta}-u$ in any closed domain $\Omega^{\circ} \subset \Omega \cap \Omega_{\delta}$, from the above formulae

$$
u_{\delta}(x)-u(x)=\mathcal{W}_{\Omega_{\delta}} \widetilde{\phi}(x)-\mathcal{W}_{\Omega} \phi(x), x \in \Omega^{\circ} .
$$

Let $\psi_{\delta}(x)=x+\delta \rho(x) \nu(x)$ and $\psi_{\delta}(x)=x+\delta \nu(x)$ be the diffeomorphisms from $\partial \Omega$ to $\partial \Omega_{\delta}$ in the case $d=2$ and in the case $d=3$ respectively. The following estimates hold.

Lemma 3.1. There exists a constant $C$ depending only on $\Omega$ such that for any function $\widetilde{\phi} \in\left(C\left(\partial \Omega_{\delta}\right)\right)^{d}$, we have

$$
\left\|\left(\mathcal{K}_{\Omega_{\delta}} \widetilde{\phi}\right) \circ \psi_{\delta}-\mathcal{K}_{\Omega}\left(\widetilde{\phi} \circ \psi_{\delta}\right)\right\|_{(\mathcal{C}(\partial \Omega))^{d}} \leq C \delta\|\widetilde{\phi}\|_{\left(\mathcal{C}\left(\partial \Omega_{\delta}\right)\right)^{d}}
$$

Proof. Fix $x \in \partial \Omega$, we then have

$$
\begin{aligned}
& \left(\mathcal{K}_{\Omega_{\delta}}^{i} \widetilde{\phi}\right) \circ \psi_{\delta}(x)-\mathcal{K}_{\Omega}^{i}\left(\widetilde{\phi} \circ \psi_{\delta}\right)(x) \\
& \quad=p \cdot v \cdot \int_{\partial \Omega}\left[-S_{i j k}(\widetilde{x}, \widetilde{y}) \widetilde{\nu}_{k}(\widetilde{y}) j_{\delta}(y)+S_{i j k}(x, y) \nu_{k}(y)\right] \widetilde{\phi}_{j} \circ \psi_{\delta} d \sigma(y)
\end{aligned}
$$

here $j_{\delta}$ denotes the Jacobian of $\psi_{\delta}$, and has the approximation $j_{\delta}(y)=1+\mathcal{O}(\delta)$.

We can express $S_{i j k}(\widetilde{x}, \widetilde{y})=S_{i j k}(\widetilde{x}-\widetilde{y})$, then due to the mean value theorem we have the following expression.

In the case $d=2$, we have

$-S_{i j k}(\widetilde{x}, \widetilde{y})=-S_{i j k}(x, y)-\delta(\rho(x) \nu(x)-\rho(y) \nu(y)) \nabla S_{i j k}(x-y+\theta(\rho(x) \nu(x)-\rho(y) \nu(y)))$, with $0<\theta<\delta$.

In the case $d=3$, we have

$-S_{i j k}(\widetilde{x}, \widetilde{y})=-S_{i j k}(x, y)-\delta(\nu(x)-\nu(y)) \nabla S_{i j k}(x-y+\theta(\nu(x)-\nu(y))), 0<\theta<\delta$.

Then combining with (2.1) and (2.5) we get

with

$$
-S_{i j k}(\widetilde{x}, \widetilde{y}) \widetilde{\nu}_{k}(\widetilde{y})=-S_{i j k}(x, y) \nu_{k}(y)+\delta T_{i j}(x, y)+\mathcal{O}(\delta),
$$

$$
\begin{array}{r}
T_{i j}(x, y)=-(\rho(x) \nu(x)-\rho(y) \nu(y)) \nabla S_{i j k}(x-y+\theta(\rho(x) \nu(x)-\rho(y) \nu(y)) \\
-S_{i j k}(x-y) \nu_{k}^{1}(y) \text { in the case } d=2 \\
T_{i j}(x, y)=-(\nu(x)-\nu(y)) \nabla S_{i j k}(x-y+\theta(\nu(x)-\nu(y))) \text { in the case } d=3 .
\end{array}
$$

Denote by

$$
T_{\Omega}^{i}\left(\widetilde{\phi} \circ \psi_{\delta}\right)=p \cdot v \cdot \int_{\partial \Omega} T_{i j}(x, y)\left(\widetilde{\phi}_{j} \circ \psi_{\delta}\right)(x) d \sigma(y),
$$


we have the equality

$$
\left(\mathcal{K}_{\Omega_{\delta}}^{i} \widetilde{\phi}\right) \circ \psi_{\delta}(x)=\mathcal{K}_{\Omega}^{i}\left(\widetilde{\phi} \circ \psi_{\delta}\right)(x)+\delta T_{\Omega}^{i}\left(\widetilde{\phi} \circ \psi_{\delta}\right)+\mathcal{O}\left(\delta^{2}\right) .
$$

By using the remarks 2.3 and 2.6 as well as the decay behavior of $S_{i j k}(x, y)$ as $|x-y| \rightarrow 0$ (as mentioned in Section 2.2), we obtain that $T_{i j}(x, y)=\mathcal{O}(\mid x-$ $\left.\left.y\right|^{-d+1}\right)$ as $|x-y| \rightarrow 0$, which implies that $T_{i j}(x, y)$ is also weakly singular. So the integral $T_{\Omega}^{i}\left(\widetilde{\phi} \circ \psi_{\delta}\right)$ is bounded on $(\mathcal{C}(\partial \Omega))^{d}$, and we have that

$$
\left\|T_{\Omega}\left(\widetilde{\phi} \circ \psi_{\delta}\right)\right\|_{(\mathcal{C}(\partial \Omega))^{d}} \leq C\left\|\widetilde{\phi} \circ \psi_{\delta}\right\|_{(\mathcal{C}(\partial \Omega))^{d}},
$$

this completes the proof.

Lemma 3.2. There exists a constant $C$ depending only on $\Omega$ and $g$ such that

$$
\left\|\widetilde{\phi} \circ \psi_{\delta}-\phi\right\|_{(\mathcal{C}(\partial \Omega))^{d}} \leq \delta C(g, \Omega) .
$$

Proof. Since the integral operator $\frac{1}{2} I+\mathcal{K}_{\Omega}$ is invertible on $(\mathcal{C}(\partial \Omega))^{d}$, combining with the trace relation (3.2), (3.4) and Lemma 1 we obtain:

$$
\begin{aligned}
\left\|\widetilde{\phi} \circ \psi_{\delta}-\phi\right\|_{(\mathcal{C}(\partial \Omega))^{d}} & \leq C\left\|\left(\frac{1}{2} I+\mathcal{K}_{\Omega}\right)\left(\widetilde{\phi} \circ \psi_{\delta}-\phi\right)\right\|_{(\mathcal{C}(\partial \Omega))^{d}} \\
\leq & C \|\left(\left(\frac{1}{2} I+\mathcal{K}_{\Omega_{\delta}} \widetilde{\phi}\right) \circ \psi_{\delta}-\left(\frac{1}{2} I+\mathcal{K}_{\Omega} \phi\right) \|_{(\mathcal{C}(\partial \Omega))^{d}}\right. \\
& +C\left\|\left(\mathcal{K}_{\Omega_{\delta}} \widetilde{\phi}\right) \circ \psi_{\delta}-\mathcal{K}_{\Omega}\left(\widetilde{\phi} \circ \psi_{\delta}\right)\right\|_{(\mathcal{C}(\partial \Omega))^{d}} \\
& \leq C\left\|g_{\delta} \circ \psi_{\delta}-g\right\|_{(\mathcal{C}(\partial \Omega))^{d}}+C \delta\|\widetilde{\phi}\|_{\left(C\left(\partial \Omega_{\delta}\right)\right)^{d}} .
\end{aligned}
$$

Due to the mean value theorem and the analyticity of $g$ in a neighborhood of $\partial \Omega$, we can easily see that there exists a constant $\mathrm{C}$ depending only on $\Omega$ and $g$ such that $\left\|g_{\delta} \circ \psi-g\right\|_{(\mathcal{C}(\partial \Omega))^{d}} \leq \delta C(g, \Omega)$.

We also have that $\|\widetilde{\phi}\|_{\left(C\left(\partial \Omega_{\delta}\right)\right)^{d}} \leq\left\|g_{\delta}\right\|_{(\mathcal{C}(\partial \Omega))^{d}}$ because of the invertibility of $\frac{1}{2}+\mathcal{K}_{\Omega_{\delta}}$.

Finally we get that $\left\|\widetilde{\phi} \circ \psi_{\delta}-\phi\right\|_{(\mathcal{C}(\partial \Omega))^{d}} \leq \delta C(g, \Omega)$.

Theorem 3.3. Let $\Omega^{\circ}$ is any closed subset of $\Omega \cap \Omega_{\delta}$, there exists a constant $C$ depending only on $g$ and $\Omega$ such that

$$
\left\|u_{\delta}(x)-u(x)\right\|_{\left(\mathcal{C}\left(\Omega^{\circ}\right)\right)^{d}} \leq \delta C(g, \Omega) .
$$

Proof. As the double-layer potential is continuous on $\Omega^{\circ}$, by using the extreme value theorem, there is a point $x_{0} \in \Omega^{\circ}$ such that

$$
u_{\delta}\left(x_{0}\right)-u\left(x_{0}\right)=\mathcal{W}_{\Omega_{\delta}} \widetilde{\phi}\left(x_{0}\right)-\mathcal{W}_{\Omega} \phi\left(x_{0}\right)=\max _{x \in \Omega^{\circ}}\left\|\mathcal{W}_{\Omega_{\delta}} \widetilde{\phi}(x)-\mathcal{W}_{\Omega} \phi(x)\right\|
$$

Recall from Section 2 that we denote $-S_{i j k}(x, y) \nu_{k}(y)$ by $\mathcal{D}_{i j}(x, y)$, we then have

$$
\begin{gathered}
u_{\delta}\left(x_{0}\right)-u\left(x_{0}\right)=\int_{\partial \Omega}\left[\mathcal{D}\left(x_{0}, \widetilde{y}\right) j_{\delta}(y) \widetilde{\phi} \circ \psi_{\delta}(y)-\mathcal{D}\left(x_{0}, y\right) \phi(y)\right] d \sigma(y) \\
=\int_{\partial \Omega} \mathcal{D}\left(x_{0}, \widetilde{y}\right)\left[j_{\delta}(y)-1\right] \widetilde{\phi} \circ \psi_{\delta}(y) d \sigma(y)+\int_{\partial \Omega} \mathcal{D}\left(x_{0}, \widetilde{y}\right)\left[\widetilde{\phi} \circ \psi_{\delta}(y)-\phi(y)\right] d \sigma(y) \\
+\int_{\partial \Omega}\left[\mathcal{D}\left(x_{0}, \widetilde{y}\right)-\mathcal{D}\left(x_{0}, y\right)\right] \phi(y) d \sigma(y):=I_{1}+I_{2}+I_{3} .
\end{gathered}
$$


It follows from Lemma 2 that

$$
I_{2} \leq \delta C_{2}(g, \Omega)
$$

By abusing of the approximation $j_{\delta}(y)=1+\mathcal{O}(\delta)$ we obtain

$$
I_{1} \leq \delta C_{1}(g, \Omega) .
$$

And tdue to the analyticity of $\mathcal{D}(x, y)$ we have that

$$
I_{3} \leq C_{3} \delta\|\phi\|_{(\mathcal{C}(\partial \Omega))^{d}} \leq \delta C_{4}(g, \Omega) .
$$

The proof is completed.

\section{DeRivation of the ASYMPtotic EXPAnsion}

Firstly, we investigate the asymptotic behavior of $\mathcal{K}_{\Omega_{\delta}} \widetilde{\phi}$ as $\delta \rightarrow 0$. Denote by $\phi_{\delta}=\widetilde{\phi} \circ \psi_{\delta}$ the vectorial function with components $\phi_{\delta, j}(j=\overline{1, d})$, by the change of variable, we can rewrite the integral $\mathcal{K}_{\Omega_{\delta}} \widetilde{\phi}$ as the following form

$$
\mathcal{K}_{\Omega_{\delta}}^{i} \widetilde{\phi}(\widetilde{x})=p \cdot v \cdot \int_{\partial \Omega}-S_{i j k}(\widetilde{x}, \widetilde{y}) \widetilde{\nu}_{k}(\widetilde{y}) \phi_{\delta, j}(x) d \sigma_{\delta}(\widetilde{y}) .
$$

Then using Taylor expansion for the kernel of double-layer potential $\mathcal{S}(\widetilde{x}, \widetilde{y})$ at $\delta=0$ when $r=x-y \neq 0$ and combining with (2.2) and (2.4), we obtain. In the case $d=2$

$$
\begin{aligned}
&-S_{i j k}(\widetilde{x}, \widetilde{y}) \widetilde{\nu}_{k}(\widetilde{y})=\underbrace{-S_{i j k}(x, y) \nu_{k}(y)}_{:=K_{i j}^{0}(x, y)}+ \\
& \sum_{n=1}^{\infty} \delta^{n} \underbrace{\left.\sum_{m+p=n} \sum_{|\alpha|=m} \frac{-\left((\rho(x) \nu(x)-\rho(y) \nu(y))^{\alpha}\right.}{\alpha !} \nabla S_{i j k}^{\alpha}(x, y)\right) \nu_{k}^{p}(y)}_{:=K_{i j}^{n}(x, y)},
\end{aligned}
$$

In the case $d=3$

$$
\begin{aligned}
&-S_{i j k}(\widetilde{x}, \widetilde{y}) \widetilde{\nu}_{k}(\widetilde{y})=\underbrace{-S_{i j k}(x, y) \nu_{k}(y)}_{:=K_{i j}^{0}(x, y)}+ \\
& \sum_{n=1}^{\infty} \delta^{n} \underbrace{\left.\sum_{m+p=n} \sum_{|\alpha|=m} \frac{-\left((\nu(x)-\nu(y))^{\alpha}\right.}{\alpha !} \nabla S_{i j k}^{\alpha}(x, y)\right) \nu_{k}^{p}(y)}_{:=K_{i j}^{n}(x, y)} .
\end{aligned}
$$

Then we introduce a sequence of integral operator $\left(\mathcal{K}_{i j}^{n} \phi_{\delta}(x)\right)_{n \in \mathbb{N}}$ defined for any $\phi_{\delta} \in(C(\partial \Omega))^{d}$ by

$$
\mathcal{K}_{\Omega}^{n, i} \phi_{\delta}(x)=p \cdot v \cdot \sum_{m+q=n} \int_{\partial \Omega} K_{i j}^{m}(x, y) \sigma^{q}(y) \phi_{\delta, j}(y) d \sigma(y), \quad n \leq 0 .
$$

Note that $\mathcal{K}_{\Omega}^{0}=\mathcal{K}_{\Omega}$. By using the same arguments as in the proof for Lemma 1, we have that the operators $\mathcal{K}_{\Omega}^{n} \phi_{\delta}$ are bounded on $(C(\partial \Omega))^{d}$. 
Theorem 4.1. Let $N \in \mathbb{N}$. There exists a constant $C$ depending on $N$ and $\Omega$ such that for any $\widetilde{\phi} \in\left(\mathcal{C}\left(\partial \Omega_{\delta}\right)\right)^{d}$,

$$
\left\|\left(\mathcal{K}_{\Omega_{\delta}} \widetilde{\phi}\right) \circ \psi_{\delta}-\mathcal{K}_{\Omega} \phi_{\delta}-\sum_{n=2}^{N} \delta^{n} \mathcal{K}_{\Omega}^{n} \phi_{\delta}\right\|_{(\mathcal{C}(\partial \Omega))^{d}} \leq C \delta^{N+1}\left\|\phi_{\delta}\right\|_{(C(\partial \Omega))^{d}}
$$

where $\phi_{\delta}:=\widetilde{\phi} \circ \psi_{\delta}$.

We now investigate the asymptotic behavior of $\mathcal{K}_{\Omega_{\delta}} \widetilde{\phi}$ as $\delta \rightarrow 0$. One can see from Theorem 2 that for each integer $N, \phi_{\delta}$ satisfies

$$
\left(\frac{1}{2} I+\mathcal{K}_{\Omega}+\sum_{n=1}^{N} \delta^{n} \mathcal{K}_{\Omega}^{n}\right) \phi_{\delta}+0\left(\delta^{N+1}\right)=g \circ \psi_{\delta} \text { on } \partial \Omega .
$$

Now using the Taylor development for $g(\widetilde{x})=\left(g_{i}(\widetilde{x})\right)_{i=\overline{1, d}}$ at $\delta=0$ we obtain the following expansions.

In the case $d=2$

$$
g_{i}(\widetilde{x})=\sum_{n=0}^{\infty} \sum_{|\alpha|=n} \frac{\delta^{n}}{\alpha !} \nabla^{\alpha} g_{i}(x)(\rho(x) \nu(x))^{\alpha}:=\sum_{n=0}^{\infty} \delta^{n} G_{i}^{n}(x)
$$

note that, the first three terms are given by

$G_{i}^{0}(x)=g_{i}(x), G_{i}^{1}(x)=\rho(x) \nu(x) \cdot \nabla g_{i}(x), G_{i}^{2}(x)=\frac{1}{2}(\rho(x) \nu(x))^{T} \nabla^{2} g_{i}(x)(\rho(x) \nu(x)) ;$

where the superscript $T$ denotes the transpose of a vector.

In the case $d=3$, we have:

$$
g_{i}(\widetilde{x})=\sum_{n=0}^{\infty} \sum_{|\alpha|=n} \frac{\delta^{n}}{\alpha !} \nabla^{\alpha} g_{i}(x)(\nu(x))^{\alpha}:=\sum_{n=0}^{\infty} \delta^{n} G_{i}^{n}(x)
$$

with the first three terms are given by

$$
G_{i}^{0}(x)=g_{i}(x), G_{i}^{1}(x)=\nu(x) \cdot \nabla g_{i}(x), G_{i}^{2}(x)=\frac{1}{2}(\nu(x))^{T} \nabla^{2} g_{i}(x)(\nu(x)) .
$$

Therefore, we obtain the following integral equation to solve

$$
\left(\frac{1}{2} I+\mathcal{K}_{\Omega}+\sum_{n=1}^{N} \delta^{n} \mathcal{K}^{n}\right) \phi_{\delta}+0\left(\delta^{N+1}\right)=\sum_{n=0}^{\infty} \delta^{n} G^{n} .
$$

We look for the solution of (4.7) in the form of power series

$$
\phi_{\delta}=\phi^{0}+\sum_{n=1}^{N} \delta^{n} \phi^{n}
$$

The equation (4.7) then can be solved recursively in the following way: Define

and for $1 \leq n \leq N$,

$$
\phi^{0}=\left(\frac{1}{2} I+\mathcal{K}\right)^{-1} G^{0}=\left(\frac{1}{2} I+\mathcal{K}\right)^{-1} g=\phi
$$

$$
\phi^{n}=\left(\frac{1}{2} I+\mathcal{K}\right)^{-1}\left(G^{n}+\sum_{p=0}^{n-1} \mathcal{K}^{n-p} \phi^{p}\right) .
$$


We obtain the following Lemma.

Lemma 4.2. Let $N \in \mathbb{N}$. There exists a constant $C$ depending only on $N$ and $\Omega$ such that

$$
\left\|\phi_{\delta}-\sum_{n=0}^{N} \delta^{n} \phi^{n}\right\|_{(C(\partial \Omega))^{d}} \leq C \delta^{N+1},
$$

where $\phi^{n}$ are defined by the recursive relation (4.9).

Now we derive the asymptotic behavior of $u_{\delta}-u$ as $\delta \rightarrow 0$. From the boundary integral representation of the solution in (3.2), after the change of variable we rewrite it as follows

$$
\mathcal{W}_{\Omega_{\delta}}^{i} \widetilde{\phi}(x)=\int_{\partial \Omega} \mathcal{D}_{i j}(x, \widetilde{y}) \phi_{\delta, j}(y) d \sigma_{\delta}(\widetilde{y})
$$

where $\phi_{\delta}:=\widetilde{\phi} \circ \psi_{\delta}$, and $\left\{\phi_{\delta, j}\right\}_{j=\overline{1, d}}$ are components of $\phi_{\delta}$.

Then due to the Taylor expansion of $S_{i j k}(x, \widetilde{y})$ with respect to $y$, combining with (2.1) and (2.5), we obtain

For the case $d=2$

$$
\begin{aligned}
&-S_{i j k}(x, \widetilde{y}) \widetilde{\nu}_{k}(\widetilde{y})=-S_{i j k}(x, y) \nu_{k}(y) \\
&+\sum_{n=1}^{\infty} \delta^{n} \underbrace{\sum_{m+p=n} \sum_{|\alpha|=m} \frac{-(\rho(y) \nu(y))^{\alpha}}{\alpha !} \nabla_{y}^{\alpha} S_{i j k}(x, y) \nu_{k}^{p}(y)}_{:=\mathcal{D}_{i j}^{n}(x, y)} .
\end{aligned}
$$

Likewise, in the case $d=3$

$$
-S_{i j k}(x, \widetilde{y}) \widetilde{\nu}_{k}(\widetilde{y})=-S_{i j k}(x, y) \nu_{k}(y)+\sum_{n=1}^{\infty} \delta^{n} \underbrace{\sum_{|\alpha|=n} \frac{-(\nu(y))^{\alpha}}{\alpha !} \nabla_{y}^{\alpha} S_{i j k}(x, y) \nu_{k}(y)}_{:=\mathcal{D}_{i j}^{n}(x, y)} .
$$

Thanks to (2.2), (2.4) and (4.12), the formula (4.10) can be rewritten as follows

$$
\begin{gathered}
\mathcal{W}_{\Omega_{\delta}}^{i}[\widetilde{\phi}](x)=\int_{\partial \Omega}\left(\mathcal{D}_{i j}(x, y)+\sum_{n=1}^{\infty} \delta^{n} \mathcal{D}_{i j}^{n}(x, y)\right) \\
\left(\phi_{j}(y)+\sum_{n=1}^{N} \delta^{n} \phi_{j}^{n}(y)+\mathcal{O}\left(\delta^{N+1}\right)\right)\left(1+\sum_{n=1}^{\infty} \delta^{n} \sigma^{n}(y)\right) d \sigma(y) \\
=\int_{\partial \Omega} \mathcal{D}_{i j}(x, y) \phi_{j}(y) d \sigma(y)+\sum_{n=1}^{N} \delta^{n} \sum_{m+k+q=n} \int_{\partial \Omega} \mathcal{D}_{i j}^{m}(x, y) \phi_{j}^{k}(y) \sigma^{q}(y) d \sigma(y)+\mathcal{O}\left(\delta^{N+1}\right) .
\end{gathered}
$$


Note that $\int_{\partial \Omega} \mathcal{D}_{i j}(x, y) \phi_{j}(y) d \sigma(y)=\mathcal{W}_{\Omega}^{i} \phi(x)$. We then define, for $n \in \mathbb{N}$ and for $x \in \Omega^{\circ}$, the terms $u_{n}=\left(u_{n}^{i}\right)_{i=\overline{1, d}}$ as follows

$$
u_{n}^{i}(x)=\sum_{m+q+k=n} \int_{\partial \Omega} \mathcal{D}_{i j}^{m}(x, y) \sigma^{q}(y) \phi_{j}^{k}(y) d \sigma(y) .
$$

We obtain that

$$
u_{\delta}-u=\sum_{n=1}^{N} \delta^{n} u_{n}(x)+\mathcal{O}\left(\delta^{N+1}\right), x \in \Omega^{\circ} .
$$

The remainder $O\left(\delta^{N+1}\right)$ depends only on $N$ and $\Omega$.

Let us compute the first order approximation of $u_{\delta}-u$ explicitly. Note that $\phi^{0}=\phi$ where $\phi$ is defined by (4.9) and

$$
\phi^{1}=\left(\frac{1}{2} I+\mathcal{K}_{\Omega}\right)^{-1}\left(G^{1}+\mathcal{K}_{\Omega}^{1} \phi\right)
$$

Therefore, in the two-dimensional case, $u_{1}=\left(u_{1}^{i}\right)_{i=\overline{1,2}}$ takes the form

$$
\begin{aligned}
u_{1}^{i}(x)=\int_{\partial \Omega} \mathcal{D}_{i j}^{1}(x, y) \phi_{j}(y) d \sigma(y)-\int_{\partial \Omega} \mathcal{D}_{i j}(x, y) & \tau(y) \rho(y) \phi_{j}(y) d \sigma(y) \\
& +\int_{\partial \Omega} \mathcal{D}_{i j}(x, y) \phi_{j}^{1}(y) d \sigma(y) .
\end{aligned}
$$

And in the three-dimensional case, $u_{1}=\left(u_{1}^{i}\right)_{i=\overline{1,3}}$ takes the form

$$
\begin{aligned}
u_{1}^{i}(x)=\int_{\partial \Omega} \mathcal{D}_{i j}^{1}(x, y) \phi_{j}(y) d \sigma(y)-2 \int_{\partial \Omega} \mathcal{D}_{i j}(x, y) H(x) \phi_{j}(y) d \sigma(y) & \\
& +\int_{\partial \Omega} \mathcal{D}_{i j}(x, y) \phi_{j}^{1}(y) d \sigma(y) .
\end{aligned}
$$

\section{REFERENCES}

[1] H. Ammari, H. Kang, M. Lim, H. ZRIBI, Conductivity interface problems. Part I: Small perturbation of an interface, Trans. Amer. Math. Soc., 362 (2010), pp. 2435-2449.

[2] R. KRESS, Linear Integral Equations, Vol. 82 2nd ed., 1999, XIV, 388 p. 1 illus.

[3] W. Borchers, W. VArnhorn, On the boundedness of the Stokes semigroup in twodimension exterior domains, Math. Zeitschrift, Volume 213, Number 1, pp. 275-299.

[4] W. VARHoRn, An explicit potential theory for the Stokes resolvent boundary value problems in three dimensions, Manuscripta Math., Volume 70, Number 1, pp. 339-361.

[5] H. Ammari, An introduction to Mathematics of Emerging Biomedical Imaging, Springer, Mathématiques et Applications, Volume 62, 2008.

[6] M. KoнR, The interior Neumann problem for the Stokes resolvent system in a bounded domain in $\mathbb{R}^{n}$, Arch. Mech., 59, 3, Warszawa 2007,pp. 283-304.

[7] E. FABES, H. KANG, J. K. SEOI, Inverse conductivity problem with one measurement: Error estimates and approximate identification for perturbed disks, SIAM J. MATH. ANAL., Vol. 30, No. 4, pp. 699-720.

[8] M. KoHR, An indirect boundary integral method for an oscillatory Stokes flow problem, IJMM, 2003:47, pp. 2916-2976.

[9] H. Ammari; P. Garapon; H. Kang; H. Lee, A method of biological tissues elasticity reconstruction using magnetic resonance elastography measurements, Quart. Appl. Math., 66 (2008), no. 1, pp. 139-175. 
[10] R.R. Coifman, A. McIntosh, and Y. Meyer, L'intégrale de Cauchy définit un opérateur bourné sur L2 pour les courbes lipschitziennes, Ann. Math., 116 (1982).

[11] G.P. GALDI, An introduction to the Mathematical Theory of the Navier-Stokes equations, Steady-State proble, Springer 2011.

[12] T. Kato, Perturbation theory for linear operators, Springer 1980.

[13] O. A. Ladyzhenskaza, The mathematics theory of viscous incompressible flow, Gordon and Breach Science Publishers 1987.

[14] Jaime H. Ortega, E. Zuazua, Generic simplicity of the eigenvalues of the Stokes sysytem in two space dimension, Advances in Differential Equations, Volume 6, Number 8, August 2001, pp. 987-1023.

[15] R. Temam, Navier-Stokes equations theory and numerical analysis, North-Holland Publishing Company Amsterdam New York Oxford 1977.

CNRS \& Laboratoire de Mathématiques (UMR 8088), Université de CergyPontoise, F-95000 Cergy-Pontoise, France.

E-mail address: thi-hong-cam.luong@u-cergy.fr

CNRS \& Laboratoire de Mathématiques (UMR 8088), Université de CergyPontoise, F-95000 Cergy-Pontoise, France.

E-mail address: christian.daveau@u-cergy.fr 